\title{
L'inactivation des deux allèles du gène BRCA1 pourrait être un facteur de bon pronostic des adénocarcinomes de l'ovaire diagnostiqués à un stade avancé
}

Les trois quarts des cancers de l'ovaire sont diagnostiqués à un stade avancé, lorsque les cellules tumorales ont déjà essaimé au-delà des ovaires et du bassin. Dans la classification de la FIGO (Fédération Internationale de Gynécologie Obstétrique), on parle de stade III en présence de nodules péritonéaux en dehors du pelvis et/ou d'adénopathies rétropéritonéales ou inguinales, et de stade IV en cas de métastases à distance (hépatiques ou pulmonaires par exemple). Le stade d'extension est le facteur pronostic essentiel du cancer de l'ovaire. Le taux de survie à 5 ans est approximativement de $25 \%$ et $10 \%$ respectivement pour les cancers de l'ovaire de stade III et IV, alors qu'il est de $80 \%$ pour les cancers de l'ovaire découverts à un stade I (maladie limitée aux ovaires). Le stade ne donne qu'une notion probabiliste du pronostic: au sein d'un groupe de patientes chez lesquelles on vient de diagnostiquer un cancer de l'ovaire à un stade avancé, nous ne disposons pas de critère morphologique ou biologique qui permettrait de reconnaître celles dont l'évolution sera finalement moins grave que le stade ne le laisse redouter.

Les femmes porteuses d'une mutation germinale altérant la fonction de BRCA1 ont un risque cumulé de cancer de l'ovaire de l'ordre de $50 \%$ $[1,2]$. Dans le modèle de Knudson $[3,4]$, les cancers survenant chez des personnes à haut risque génétique sont a priori du même type que les cancers sporadiques car les anomalies génétiques sont similaires dans les deux cas. La prédisposition génétique au cancer est due, dans l'hypothèse de Knudson, à une mutation constitutionnelle dont la caractéristique est d'être identique à l'une des mutations acquises participant à la genèse des cancers sporadiques.

En accord avec l'hypothèse de Knudson, des mutations acquises altérant le gène BRCA1 ont été décrites dans des cancers de l'ovaire sporadiques. Cependant, si des pertes de matériel génétique sont fréquentes au locus de BRCA1 dans les cancers sporadiques (entraînant l'inactivation d'un seul des deux allèles du gène), c'est seulement dans une petite minorité des cancers sporadiques $(\approx 10 \%)$ que les deux allèles de BRCA1 sont inactivés [5]. Cette faible proportion contraste avec une fréquence d'inactivation des deux allèles de BRCA1 proche de $100 \%$ dans les cancers survenus chez les femmes porteuses d'une mutation germinale BRCA1. Au regard des mutations de BRCA1 dans le cancer de l'ovaire, l'hypothèse de Knudson est donc vraie, avec cependant une nuance de taille: $10 \%$ seulement des cancers sporadiques ont la même anomalie des deux allèles de BRCA1 que celle observée dans les formes germinales.

\section{Une étude épidémiologique rétrospective récemment publiée}

Le but de l'étude était de préciser les caractéristiques cliniques et histologiques des cancers de l'ovaire chez les femmes porteuses d'une mutation germinale de BRCA1 [1]. Cette étude rétrospective a porté sur 53 cas de cancer de l'ovaire diagnostiqué chez des femmes porteuses d'une mutation germinale de BRCA1 (mutation identifiée par biologie moléculaire, ou bien déduite de l'analyse de l'arbre généalogique de la patiente). Chacun de ces cas a été apparié à un cas de cancer de l'ovaire sporadique. L'appariement a été réalisé sur l'âge, le type histologique, le grade et le stade. Le groupe témoin a été tiré d'une base de données dans laquelle aucun sujet n'avait d'antécédent évocateur d'une prédisposition héréditaire au cancer. La principale conclusion de cette étude est que les cancers de l'ovaire diagnostiqués chez les femmes ayant une mutation germinale de BRCA1 semblent être de bien meilleur pronostic que les cancers de l'ovaire sporadiques. La médiane de survie actuarielle est de 77 mois dans le groupe de patientes atteintes d'un cancer de l'ovaire de stade III $(n=38)$ ou IV $(n=5)$ porteuses d'une mutation germinale de $B R C A 1$, alors que cette médiane de survie n'est que de 29 mois dans le groupe témoin $(\mathrm{p}<0,001)$.

Un fait déjà décrit mais encore peu connu est également rapporté dans cette étude: la proportion de carcinomes de type séreux est plus élevée parmi les cancers de l'ovaire associés à une mutation germinale de $B R C A 1$ que dans les cancers sporadiques (80\% contre $50 \%)$.

Analyse critique de cette étude

De nombreux biais de sélection pourraient artificiellement faire 
paraître la proportion de rémission prolongée plus importante chez les patientes porteuses d'une mutation germinale de BRCA1. La sélection des femmes testées pour une mutation germinale de BRCA1 pourrait par exemple être biaisée par la disponibilité de matériel et l'étude moléculaire entreprise uniquement chez des patientes vivantes. Les auteurs semblent avoir pris soin d'éviter ce biais grossier en incluant dans leur étude toutes les personnes susceptibles d'avoir été porteuses d'une mutation germinale de $B R C A 1$, y compris les patientes décédées. Bien entendu, ce biais peut ne pas avoir été totalement éliminé. Aucun autre biais évident ne paraissant susceptible de fausser les résultats de cette étude, l'observation d'une survie prolongée des cancers de l'ovaire associés à une mutation germinale de BRCA1 pourrait donc correspondre à un fait authentique.

Hypothèses sur les altérations acquises de BRCA1 et le pronostic des cancers de l'ovaire

L'hypothèse des auteurs de l'article est que la différence de pronostic entre les formes de cancers de l'ovaire associées à des mutations germinales de BRCA1 et les formes sporadiques pourrait reposer sur des différences de profils de mutations somatiques dans des gènes autres que BRCA1.

On peut, peut-être, compléter l'hypothèse des auteurs en supposant qu'il existe deux types de combinaison d'événements génétiques à l'origine des cancers de l'ovaire: une mutation d'un allèle BRCA1 coopérant avec une autre anomalie génétique inconnue, à l'origine des cancers de mauvais pronostic dans $90 \%$ des formes sporadiques. Une inactivation des deux allèles de BRCA1 dans les formes germinales et dans $10 \%$ des formes sporadiques, donnant des tumeurs de meilleur pronostic. Cette dernière hypothèse devrait être facile à tester, puisqu'elle implique un pronostic meilleur des cancers sporadiques avec deux plutôt qu'avec un allèle $B R C A 1$ muté... à suivre.
1. Rubin S, Benjamin I, Behbakht K, et al. Clinical and pathological features of ovarian cancer in women with germ-line mutations of BRCA1. N Eng J Med 1996; 335 : 1413-6.

2. Easton D, Ford D, Bishop T, et al. Breast and ovarian cancer incidence in BRCA 1-mutation carriers. Am J Hum Genet 1995; 56: 265-71.

3. Knudson AG. Mutation and cancer: a statistical study of retinoblastoma. Proc Natl Acad Sci 1971; 68: 820-3.

4. Bonaïti-Pellié C, Feingold J. Rétinoblastome: de l'épidémiologie génétique à la biologie moléculaire. Med Sci 1990; 6: 972-9.

5. Merajver S, Pham T, Caduff R, et al. Somatic mutations in the BRCA1 gene in sporadic ovarian tumours. Nature Genet 1995; 9 : 439-43.

Soins et Santé Demain: Vers une santé hors murs, Lyon, France, 8-10 décembre 1997.

Cette réunion $s$ 'inscrit dans le cadre des 10es Entretiens Jacques Cartier.

Contact: Betty Dodet, Fondation Marcel-Mérieux, 17, rue Bourgelat, BP 2021, 69227 Lyon Cedex 02, France. Fax: (33) 72737993

E-mail : 100765.1401@Compuserve.Com

\section{Institut Curie Remise du Prix Jeanne Loubaresse institut Curie 1995 La Science au Service de l'Homme À LAURENT DEgos ET ANNE DEJEAN-ASSÉMAT}

Le Prix Jeanne Loubaresse - Institut Curie a été remis le mardi 4 février 1997 par le Professeur Jean Bernard, membre de l'Académie des Sciences, à Madame Anne Dejean-Assémat et à Monsieur Laurent Degos lors d'une cérémonie qui s'est déroulée à l'Institut Curie.

Ce prix, d'un montant de 350000 F, a été décerné par le Conseil d'Administration de l'Institut Curie du 15 février 1996 , sur proposition d'un jury présidé par Monsieur Roger Monier et composé de Messieurs Philippe Ascher, Thierry BoonFalleur, Pierre Corvol, Jacques Demaille, Dominique Maraninchi, Bernard Roques, Gérard Toulouse et Gilbert Weill.

Il récompense Monsieur Laurent Degos, Professeur d'Hématologie à l'hôpital Saint-Louis (Université Paris 7) et Madame Anne Dejean-Assémat, Directeur de recherche à l'Inserm (Institut Pasteur), pour avoir montré qu'un dérivé de la vitamine $A$, l'acide « tout trans rétinö̈que », provoque des rémissions complètes dans une forme particulière de leucémie, la leucémie aiguë à promyélocytes, et pour avoir identifié l'anomalie génétique caractéristique de cette leucémie. 